\title{
Value of direct radionuclide cystography in diagnosing vesico-peritoneal fistulae
}

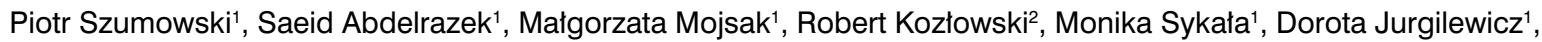
Janusz Myśliwiec

'Department of Nuclear Medicine, Medical University of Bialystok, Poland

${ }^{2}$ Department of Urology, J. Sniadecki Provincial Hospital of Bialystok, Poland

[Received 28 XII 2016; Accepted 25 IV 2017]

\begin{abstract}
A 36-year-old female patient underwent a laparoscopic surgery to remove a uterine fibroid. The procedure failed to relieve the pelvic pain, although its nature changed. After a period of observation, the patient was re-admitted to hospital on suspicion of a vesico-uterine fistula to be differentiated with endometriosis. Diagnostic investigations - cystography, cystoscopy, computed tomography and magnetic resonance — did not reveal a fistula. Laparoscopy was performed, with a possible biopsy in order to eliminate endometriosis. The result was negative, but chronic progressive reactive/inflammatory lesions were noticed, possibly indicating the presence of a vesico-peritoneal fistula. Therefore, a direct radionuclide cystography was performed. The scintigraphic images single-photon emission computed tomography (SPECT/CT) showed a radioactive spot, indicative of a vesico-peritoneal fistula. The fistula was treated for three months by catheterisation of the urinary bladder. The follow-up SPECT-CT did not reveal any urine leakage from the bladder. The clinical symptoms disappeared as well.
\end{abstract}

KEY words: radionuclide cystography, single-photon emission computed tomography, vesico-peritoneal fistule

Nucl Med Rev 2017; 20, 2: 105-106

\section{Introduction}

Direct radionuclide cystography (DRC) is one of the most commonly used methods of diagnosing vesico-ureteral reflux, particularly in children [1]. The technique of the analysed investigation (use of a gamma camera to measure ionising radiation emitted by radiolabelled saline solution instilled directly into the bladder) allows for a wider application of the method. DRC can also be useful in diagnosing vesical fistulae, as is exemplified by the discussed case.

\section{Case report}

A 36-year-old female patient underwent a laparoscopic surgery to remove a uterine fibroid. The operation was performed because the patient had intermittently suffered from relatively strong burning, stinging pain in the suprapubic pelvic region, as well as to eliminate the risk (described in the computed tomography - CT examination) of pressure on the small intestine, which could im-

Correspondence to: Piotr Szumowski, PhD

Department of Nuclear Medicine, Medical University of Bialystok

M. Skłodowskiej-Curie St. 24A, 15-276 Bialystok, Poland

Tel.: + 48857468770

Fax: +48 857468771

E-mail: piotrmjs@wp.pl pair intestinal passage. During the surgery, the uterine tumour was removed and the adhesions between the uterus and the urinary bladder were separated. The procedure failed to relieve the pelvic pain, although its nature changed (it became particularly intense when the bladder was full, before and immediately after micturition). After a period of observation lasting approximately 6 months, the patient was re-admitted to hospital on suspicion of a vesico-uterine fistula to be differentiated with endometriosis. Two diagnostic investigations - cystography and cystoscopy — did not reveal a fistula. Since the clinical symptoms persisted, a CT scan and an magnetic resonance (MR) with contrast were performed. The MR identified a small, $5 \times 7 \times 9 \mathrm{~mm}$, fluid collection trapped in adhesions located outside the urinary bladder, among the intestinal loops. Another laparoscopy was performed, with a possible biopsy in order to eliminate endometriosis. The result was negative, but chronic progressive reactive/inflammatory lesions were noticed, possibly indicating the presence of a vesico-peritoneal fistula. Therefore, a DRC was performed. The urinary bladder was filled with $650 \mathrm{~mL}$ of saline solution and technetium-99m diethylene triamine pentaacetate (DTPA-99mTc) through a Foley catheter. Next, single-photon emission computed tomography (SPECT-CT) of the urinary bladder was conducted by using gamma-camera. The scintigraphic images showed a radioactive spot located on the left and adjoining the posterior wall of the urinary bladder, indicative of a vesico-peritoneal fistula. Among the intestinal loops, another radioactive spot was revealed, not connected with the urinary bladder, which suggested the 

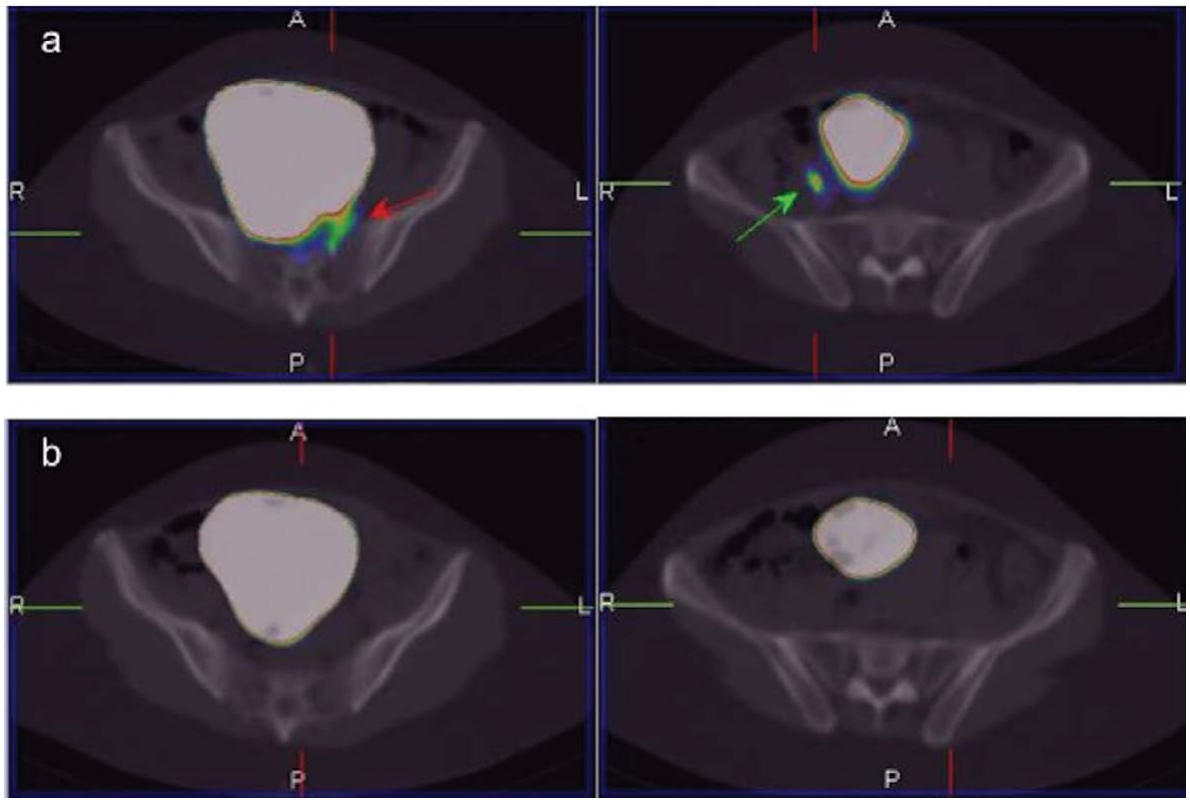

Figure 1. A. SPECT-CT direct radionuclide cystography, following instillation of $650 \mathrm{~mL}$ of DTPA-99mTc labelled saline solution, reveals, on the left side, a perivesical radioactive area adjoining the posterior wall of the urinary bladder, i.e. a vesico-peritoneal fistula; below: radioactive urine pool among the intestinal loops, not connected with the urinary bladder; B. Normal SPECT-CT direct radionuclide cystography image after treatment; SPECT-CT — single-photon emission computed tomography; DTPA-99mTc — technetium-99m diethylene triamine pentaacetate

presence of a small amount of urine in that area (Figure 1A). The fistula was treated for three months by catheterisation of the urinary bladder. The follow-up SPECT-CT did not reveal any urine leakage from the bladder or any free fluid in the peritoneal cavity (Figure 1B). The clinical symptoms disappeared as well.

\section{Discussion}

The above case confirms the usefulness of the DRC method for diagnosing vesico-peritoneal fistulae, especially small-to-moderate ones. This is connected with the high sensitivity of radioisotope imaging techniques. These methods make it possible to detect substances whose quantities are expressed in picomoles, whereas other imaging tests (cystography, CT, or even MR) are only capable of identifying millimolar amounts of substances [2,3]. Furthermore, the use of hybrid devices, i.e. a gamma camera combined with a CT scanner makes it possible to superimpose one type of image over the other, which significantly improves the precision of the anatomical localisation of a given lesion (in this case, the location of urine leakage and free urine in the pelvis). It should also be emphasised that the absorbed dose of ionising radiation received by the patient in the course of the examination is relatively low $(0.6 \mathrm{mSv}$ on average), lower than in the case of cystography (1 mSv on average) [4].

\section{Conclusion}

DRC can be useful method for diagnosing vesico-peritoneal fistulae, especially small-to-moderate ones.

\section{Conflict of interest}

The authors declare that they have no conflict of interest.

\section{References}

1. Fettich J, Colarinha P, Fischer S, et al. Guidelines for direct radionuclide cystography in children. Eur J Nucl Med Mol Imaging. 2003; 30(5): B39-B44, doi: 10.1007/s00259-003-1137-x, indexed in Pubmed: 12692686.

2. Yu NC, Raman SS, Patel M, et al. Fistulas of the genitourinary tract: a radiologic review. Radiographics. 2004; 24(5): 1331-1352, doi: 10.1148/rg.245035219, indexed in Pubmed: 15371612.

3. Tang YZ, Booth TC, Swallow D, et al. Imaging features of colovesical fistulae on MRI. Br J Radiol. 2012; 85(1018): 1371-1375, doi: 10.1259/bjr/55871151, indexed in Pubmed: 22674706.

4. Andersson M, Johansson L, Minarik D, et al. Effective dose to adult patients from 338 radiopharmaceuticals estimated using ICRP biokinetic data, ICRP/ICRU computational reference phantoms and ICRP 2007 tissue weighting factors. EJNMMI Phys. 2014; 1(1): 9, doi: 10.1186/2197-7364-1-9, indexed in Pubmed: 26501451. 